\title{
A Two-stage Sensing Technique for Dynamic Spectrum Access
}

\author{
Ling Luo and Sumit Roy \\ Dept. of Electrical Engineering \\ University of Washington, Seattle, USA, 98195
}

\begin{abstract}
Dynamic spectrum access (DSA) is a promising approach for mitigating spectrum scarcity. Underlying DSA is the need for fast and reliable spectrum sensing over a potentially large band. In [4], the concept of two-stage sensing scheme was introduced. In this work, we develop models for performance analysis based on mean time to detect an idle channel. Simulation results show that two-stage sensing leads to faster detection than conventional single-stage random search. System-level issues such as the impact of bandwidth of coarse sensing block and sensing duration of energy detector on mean detection time are also explored.
\end{abstract}

\section{INTRODUCTION}

Recent studies show that licensed spectrum (especially TV bands) is used inefficiently generically [1][2], i.e., about $52 \%$ of TV channels in Seattle area [3] are unused, constituting 'white spaces'. Dynamic spectrum access (DSA) has thus been proposed as a means to improve spectral efficiency by opening it for use by (new) secondary users on a non-interfering basis with the primary users. The key to enabling this is fast, effective detection of idle channels by secondary users, as characterized by mean time to detection of an idle channel.

The average time to successful spectrum sensing naturally depends on the search algorithm, which can be broadly classified into a) random and b) deterministic approaches [5][6]. Since the performance of this sub-system is fundamental to many other performance aspects of cognitive networks, recent attention has focussed on innovations to the core sensing subsystem. As argued in [13], jointly adapting key link and MAC layer algorithms to the environment is a promising approach. However, another source of innovation that is relatively underexplored is architectural in nature. Our work presents a contribution along these lines by exploiting two stages of sensing Coarse Resolution Sensing $(C R S)$ and Fine Resolution Sensing $(F R S)$.

In two-stage sensing, the total system bandwidth is first divided into several equal-size coarse sensing blocks (CSB). The CSB with(without) idle channels in it is denoted as $\operatorname{CSBW}(C S B N)$ in this paper. The first stage of Coarse Resolution Sensing is performed to locate a CSB with idle channels; this is followed by fine resolution sensing, shown in Fig.1(b). Once FRS fails in detecting all the idle channels within CSBW, the search scheme will return to CRS. The analytical and simulation results of two-stage sensing in terms of mean detection time are provided in the rest of this paper. Moreover, trade-offs among bandwidth of coarse sensing step, sensing duration and the overall detection time are also explored.

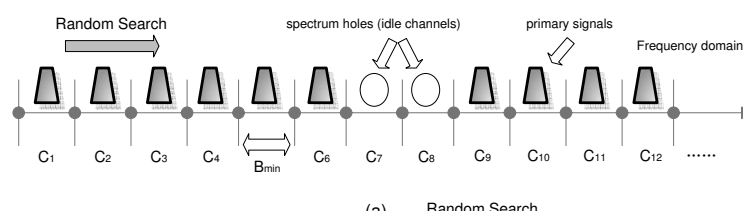

(a) Random Search

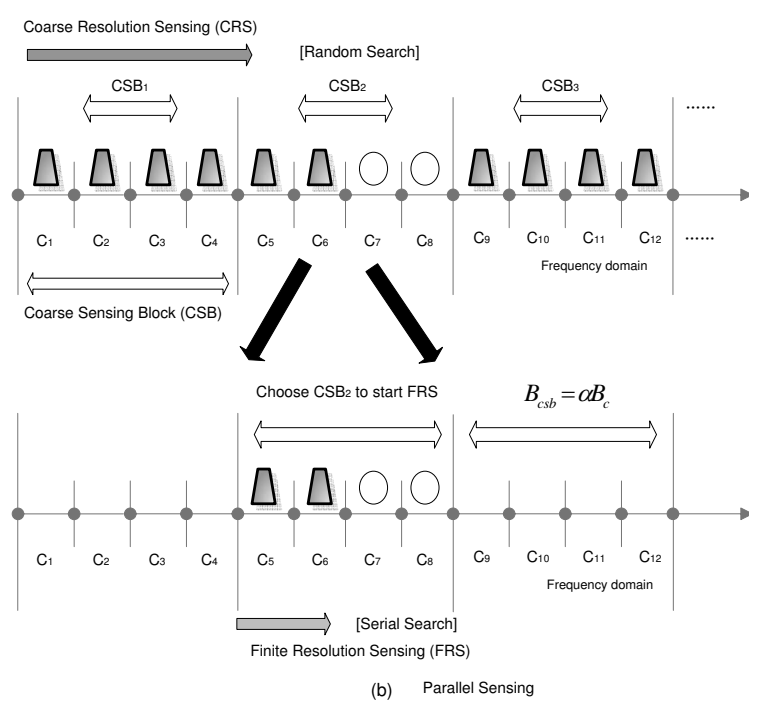

Fig. 1. Channel Model and Sensing Scheme

\section{SySTEM DESCRIPTION}

\section{A. System Model}

We assume that the entire spectral band is composed of an $N$-set of contiguous discrete frequency domain channels with equal-size bandwidth $B_{c}$, which is equal to the bandwidth of a primary signal. Let $L$ be the number of idle channels (unoccupied by primary users), where typically $L / N \ll 1$. We assume that the $L$ idle channels are randomly scattered over the $N$-set. The binary variable $O_{k}$ will be used to denote the status of the $k$-th channel, where $O_{k}=0(1)$ means that channel is busy (idle). Hence

$$
P\left(O_{k}=1\right)=\frac{L}{N}, k=1,2,3 \ldots N
$$

All channels are assumed to be AWGN with normalized amplitude gains of unity. We assume that the simplest and most 
widespread - i.e., noncoherent square-law or energy detection - is used. The observed signal samples are filtered to a detector bandwidth ( $\left.B_{\text {sense }}\right)$, passed through a square law detector, and integrated over a sensing duration before comparing with a decision threshold $\left(D_{t}\right)$. We denote $P_{n}$ and $P_{s i g}$ as the noise power and power of the primary signal observed by the secondary user, which are given by

$$
\begin{aligned}
P_{n} & =k T B_{\text {sense }} \\
P_{\text {sig }} & =\bar{\gamma} P_{n}
\end{aligned}
$$

where $k$ is the boltzmann constant $\left(1.38 \times 10^{-23} \mathrm{~J} / \mathrm{k}\right), T$ is the system temperature (300 K), and $\bar{\gamma}$ is the signal-to-noise ratio (SNR).

\section{B. Channel Sensing}

Two conventional search schemes - random and serial search, are widely used in channel sensing. As shown in Fig.1(a), the secondary user randomly selects a channel; if the channel is detected to be busy, the user then picks another channel randomly; the process terminates when an idle channel is found. In serial search, the secondary user searches channels in sequence beginning from an initial starting point till an idle channel is discovered. Both of two schemes can be defined as 1-stage sensing scheme.

However, for small $L / N$ [6] showed that the detection performance for both schemes is inadequate, indicating the need for better approaches. A novel multi-resolution approach was proposed in [4] for nodes equipped with multiple antennas, that allowed parallel (simultaneous) scanning of disjoint frequency bands to improve the mean time to detection. We adapt the idea for a two-stage sensing scheme for one-antenna nodes. As shown in Fig.1(b), the total spectrum is divided into $\beta$ channel sensing blocks (CSB), each of which contains $\alpha$ channels of width $B_{c}\left(\alpha=N / \beta, B_{c s b}=\alpha B_{c}\right)$. The sensing strategy is then composed of two stages: coarse (CRS) and fine (FRS) sensing. In the former, the first CSB with an idle channel is located; thereafter, fine sensing detects the idle channel within the block. In CRS, random search is used for detection with sensing bandwidth equal to a coarse sensing block $\left(B_{\text {sense }}=B_{c s b}\right)$. On the other hand, FRS uses serial search within a CSB, where $B_{\text {sense }}=B_{c}$. In FRS, a false alarm will result in a penalty equal to $J$ sensing durations to recover from the error and resume scanning. Further, if no idle channel is detected after FRS, the device will re-initialize to CRS as shown in Fig.2.

\section{Analysis of Detection and False Alarm Probability in Two-STAge Sensing}

\section{A. Detection and False Alarm in FRS}

In FRS, the detector bandwidth is equal to the sensing bandwidth $\left(B_{\text {sense }}=B_{c}\right)$. Further, the decision process chooses between one of two hypotheses: $H_{1}$ (noise only) denoting primary signal absence and $H_{0}$ where the primary signal is present. [6] has shown the output (denoted as $x$ ) of

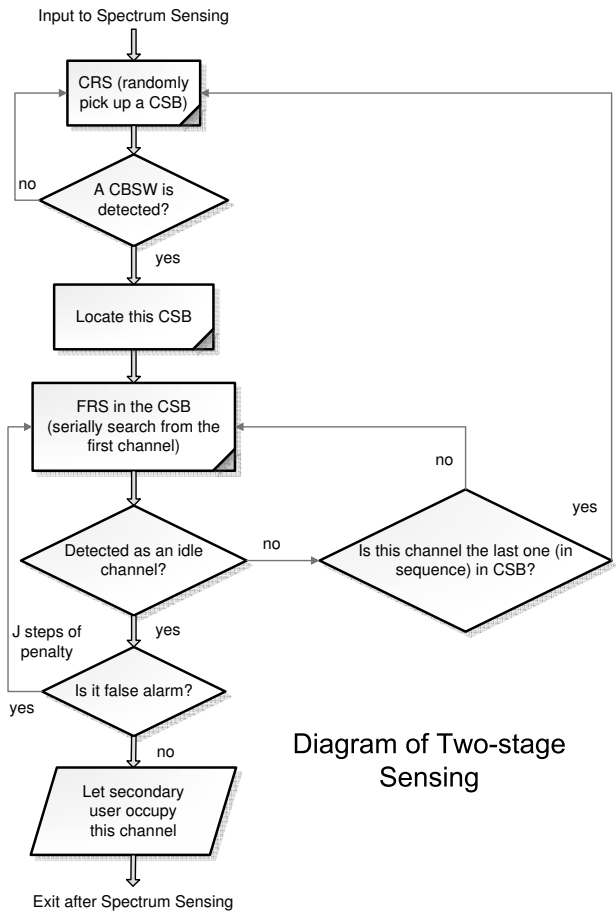

Fig. 2. Diagram of Two-stage Sensing

the device as below

$$
\begin{aligned}
& f\left(x \mid H_{1}\right)=\frac{1}{\sqrt{2 \pi} \sigma_{n}} \exp \left(-\frac{x^{2}}{2 \sigma_{n}^{2}}\right) \\
& f\left(x \mid H_{0}\right)=\frac{1}{\sqrt{2 \pi} \sigma_{n}} \exp \left(-\frac{\left(x-\sqrt{P_{\text {sig }}}\right)^{2}}{2 \sigma_{n}^{2}}\right)
\end{aligned}
$$

where $\sigma_{n}=\sqrt{P_{n} / 2}=\sqrt{k T B_{c} / 2}$ and $x$ represents the power of an observed sample.

Let $z$ denote the final decision variable upon time integration: $z=\sum_{i=1}^{M_{f}} x_{i}^{2}$, where $M_{f}=\left\lfloor B_{c} \cdot T_{f r s}\right\rfloor(\lfloor x\rfloor$ is the largest integer contained in $x$; $T_{f r s}$ is the integration time by noncoherent detector in FRS). It is clear that $z$ follows chi-square distribution under $H_{1}$ and non-central chi-square distribution under $H_{0}$, both with $M_{f}$ degrees of freedom [12]. The detection probability $\left(P f_{d}\right)$ and false alarm probability $\left(P f_{f a}\right)$ respectively correspond to the event that a secondary user successfully detects the idle channel under $H_{1}$ and claims that no primary signal is present under $H_{0}$. Thereby $P f_{d}$ and $P f_{f a}$ of the noncoherent detector in FRS can be expressed as [10]

$$
\begin{aligned}
P f_{d} & =F_{z \mid H_{1}}\left(D_{t} / \sigma_{n}^{2}\right)=\frac{\gamma\left(M_{f}, D_{t} / 2 \sigma_{n}^{2}\right)}{\Gamma\left(M_{f}\right)} \\
P f_{f a} & =F_{z \mid H_{0}}\left(D_{t} / \sigma_{n}^{2}\right)=1-Q_{M_{f}}\left(\sqrt{\bar{\gamma} M_{f}}, \sqrt{D_{t} / \sigma_{n}^{2}}\right)
\end{aligned}
$$

where $\Gamma$ and $\gamma$ are respectively gamma function and lower incomplete gamma function $\Gamma(M)=\int_{0}^{\infty} t^{M-1} e^{-t} d t$, $\gamma(\alpha, x)=\int_{0}^{x} t^{\alpha-1} e^{-t} d t ; Q_{m}$ is the generalized Marcum Qfunction $Q_{m}(a, b)=\int_{b}^{\infty} \frac{x^{m}}{a^{m-1}} e^{-\frac{x^{2}+a^{2}}{2}} I_{m-1}(a x) d x$. 


\section{B. Detection and False Alarm in CRS}

The analysis of CRS sensing stage is more complex. The decision process chooses between one of two hypotheses: $H_{1}^{\prime}$ when there exists at least one idle channel in the CSB and $H_{0}^{\prime}$ when there is none. If hypothesis $H_{1}^{\prime}$, FRS stage is invoked to precisely identify an idle channel contained in the CSB. In our i.i.d model, a primary signal occupies a discrete channel with probability $1-\frac{L}{N} ; L$ idle channels are then randomly scattered over the $N$-set of channels. Therefore, the number of idle channels $n$ in a CSB follows a binomial distribution $(n \sim B(\alpha, L / N))$. As a sub-hypothesis of $H_{1}^{\prime}, H_{1,(k)}^{\prime}$ denotes the event that there are exactly $k$ idle channels $(1 \leq k \leq \alpha)$ in a CSB; hence

$$
\begin{aligned}
\operatorname{Pr}\left(H_{1,(k)}^{\prime}\right) & =\operatorname{Pr}(n=k)=\left(\begin{array}{c}
\alpha \\
k
\end{array}\right)\left(\frac{L}{N}\right)^{k}\left(1-\frac{L}{N}\right)^{\alpha-k} \\
\operatorname{Pr}\left(H_{1}^{\prime}\right) & =1-\operatorname{Pr}(n=0)=1-\left(\frac{N-L}{N}\right)^{\alpha} \\
\operatorname{Pr}\left(H_{1,(k)}^{\prime} \mid H_{1}^{\prime}\right) & =\frac{\operatorname{Pr}(n=k)}{\operatorname{Pr}\left(H_{1}^{\prime}\right)}=\left(\begin{array}{c}
\alpha \\
k
\end{array}\right) \frac{L^{k}(N-L)^{\alpha-k}}{N^{a}-(N-L)^{a}}
\end{aligned}
$$

The p.d.f of the measurements $y$ that are input to the CRS detector, conditional on the hypothesis, are thus given by

$$
\begin{aligned}
f\left(y \mid H_{1,(k)}^{\prime}\right) & =\frac{1}{\sqrt{2 \pi} \sigma_{n}^{\prime}} \exp \left(-\frac{\left(x-(\alpha-k) \sqrt{P_{\text {sig }}}\right)^{2}}{2{\sigma_{n}^{\prime}}^{2}}\right) \\
f\left(y \mid H_{0}^{\prime}\right) & =\frac{1}{\sqrt{2 \pi} \sigma_{n}^{\prime}} \exp \left(-\frac{\left(x-\alpha \sqrt{P_{\text {sig }}}\right)^{2}}{2{\sigma_{n}^{\prime}}^{2}}\right)
\end{aligned}
$$

For the noncoherent detector in CRS, the noise power at the detector input $\sigma_{n}^{\prime}=\sqrt{P_{n}^{\prime} / 2}=\sqrt{\alpha P_{n} / 2}$. Thereby, the noise power is amplified by factor $\alpha$ relative to FRS scenario, while the signal power remains the same. Assuming the the detector uses $M_{c}$ samples, the decision statistic is $z=\sum_{i=1}^{M_{c}} y_{i}^{2}, M_{c}=\left\lfloor B_{c s b} \cdot T_{c r s}\right\rfloor$ where $T_{c r s}$ is the integration time in CRS. Therefore, detection probability $P c_{d}$ and false alarm probability $P c_{f a}$ in CRS are given by

$$
\begin{aligned}
P c_{d} & =F_{z \mid H_{1}^{\prime}}\left(D_{t}^{\prime} /{\sigma_{n}^{\prime}}^{2}\right)=\sum_{k=1}^{\alpha} F_{z \mid H_{(k)}^{\prime}}\left(D_{t}^{\prime} /{\sigma_{n}^{\prime}}^{2}\right) \operatorname{Pr}\left(H_{(k)}^{\prime} \mid H_{1}^{\prime}\right) \\
& =\sum_{k=1}^{\alpha} \operatorname{Pr}\left(H_{1,(k)}^{\prime} \mid H_{1}^{\prime}\right)\left[1-Q_{M_{c}}\left(\sqrt{\frac{\alpha-k}{\alpha} \bar{\gamma} M_{c}}, \sqrt{\frac{D_{t}^{\prime}}{\alpha \sigma_{n}^{2}}}\right)^{P}\right. \\
P c_{f a} & =F_{z \mid H_{0}^{\prime}}\left(D_{t}^{\prime} /{\sigma_{n}^{\prime}}^{2}\right)=1-Q_{M_{c}}\left(\sqrt{\bar{\gamma} M_{c}}, \sqrt{D_{t}^{\prime} / \alpha \sigma_{n}^{2}}\right)
\end{aligned}
$$

\section{Mean Detection Time}

Each channel scanning step has two components: $T_{s}$ and $T_{i}$. $T_{s}$, assumed to be a constant, is the fixed duration required for the receiver to switch its sensing circuitry to a new channel, depending on the circuit implementation. $T_{i}$ is the integration time for noncoherent detector to reach a decision on channel status (busy/idle), and hence is a function solely of the detector configuration and the desired $P_{d^{-}} P_{f a}$.

The overall metric - mean detection (acquisition) time $\left(\overline{T_{d e t}}\right)$ to successfully acquire an idle channel for a secondary user
- can be written as a function of two components mentioned above:

$$
\overline{T_{d e t}}=\overline{S_{d e t}} \cdot\left(T_{s w}+T_{i}\right)
$$

[6] has already analyzed mean number of detection steps for conventional random and serial search, i.e.,

$$
\begin{aligned}
\overline{S_{\text {ran }}} & =\frac{(N-L) J P_{f a}+N}{P_{d} L}=\frac{\left(1-\frac{L}{N}\right) J P_{f a}+1}{P_{d} \frac{L}{N}} \\
\overline{S_{\text {ser }}} & =\frac{(N-L) J P_{f a}+N}{P_{d}(L+1)}
\end{aligned}
$$

Thus, in the ideal scenario $\left(P_{d}=1, P_{f a}=0\right)$ these simplify to

$$
\overline{S_{\text {ran }, \text { ideal }}}=N / L, \overline{S_{\text {ser, ideal }}}=N /(L+1)
$$

Using the above, the average number of detection steps for two-stage sensing is provided next.

\section{A. Analysis of Two-Stage Sensing}

Because two-stage sensing has two stages - CRS and FRS, the mean number of detection steps can be written as

$$
\overline{S_{d e t}}=\overline{S_{c r s}}+\overline{S_{f i n}}
$$

In the i.i.d model, each CSB has the same probability of $\operatorname{Pr}\left(H_{1}^{\prime}\right)$ to contain a white space. Similar to the analysis of conventional random search, the mean number of steps for CRS to successfully detect a white space in a CSB can be written as

$$
\overline{S_{c r s, d e t}}=1 /\left(\operatorname{Pr}\left(H_{1}^{\prime}\right) P c_{d}\right)
$$

However, CRS will be re-initialized by missed detection of idle channels in the following FRS, which occurs with probability

$$
\begin{aligned}
P_{\text {miss }} & =\sum_{k=1}^{\alpha} \operatorname{Pr}\left(H_{(k)}^{\prime} \mid H_{1}^{\prime}\right)\left(1-P f_{d}\right)^{k} \\
& =\sum_{k=1}^{\alpha}\left(\begin{array}{c}
\alpha \\
k
\end{array}\right) \frac{L^{k}(N-L)^{\alpha-k}}{N^{a}-(N-L)^{a}}\left(1-P f_{d}\right)^{k}
\end{aligned}
$$

The number of steps for such missed detection follows a geometric distribution, with the expected value of $1 /(1-$ $\left.P_{\text {miss }}\right)$.Thus the average number of steps in CRS stage is given

$$
\begin{aligned}
\overline{S_{c r s}} & =\overline{S_{c r s, d e t}} /\left(1-P_{\text {miss }}\right) \\
& =\frac{1 /\left[\left(1-\left(\frac{N-L}{N}\right)^{\alpha}\right) P c_{d}\right]}{1-\sum_{k=1}^{\alpha}\left(\begin{array}{c}
\alpha \\
k
\end{array}\right) \frac{L^{k}(N-L)^{\alpha-k}}{N^{a}-(N-L)^{a}}\left(1-P f_{d}\right)^{k}}
\end{aligned}
$$

The analysis of FRS stage must be divided into two conditional events: a) after correct detection and b) after false alarm in CRS stage ( $\overline{S_{f r s, c o r}}$ and $\left.\overline{S_{f r s, f a l}}\right)$, respectively. Assuming that exactly $i$ idle channels exist in a CSB (i.e, $n=i$ ), the mean number of steps by FRS is given by [6]

$$
E\left[S_{f r s, c o r} \mid n=i\right]=\frac{(\alpha-i) J P f_{f a}+\alpha}{P f_{d} \cdot i}
$$


$\overline{S_{f r s, c o r}}=\sum_{i=1}^{\alpha}\left(\begin{array}{c}\alpha \\ i\end{array}\right) \frac{L^{i}(N-L)^{\alpha-i}}{N^{a}-(N-L)^{a}} \frac{(\alpha-i) J P f_{f a}+\alpha}{P f_{d} \cdot i}$

For each false alarm in CRS, the subsequent FRS will result in $\left(1+J P_{f a}\right) \alpha$ more steps on average before discovery. Because the probability of CSB with atleast one idle channel is $\operatorname{Pr}\left(H_{1}^{\prime}\right)$, the mean number of steps caused by false alarm in CRS can be written as

$$
\begin{aligned}
\overline{S_{f r s, f a l}} & =\left(1-\operatorname{Pr}\left(H_{1}^{\prime}\right)\right) P c_{f a} \overline{S_{c r s, d e t}}\left(1+J P f_{f a}\right) \\
& =\frac{P c_{f a} \alpha\left(1+J P f_{f a}\right)(N-L)^{k}}{P c_{d}\left(N^{k}-(N-L)^{k}\right)}
\end{aligned}
$$

Hence the expected number of steps for FRS sensing stage $\left(\overline{S_{f r s}}\right)$, is shown in (16) on the top of next page.

In the ideal scenario $\left(P f_{d}=P c_{d}=1, P f_{f a}=P c_{f a}=0\right)$, the overall mean number of detection steps simplifies to

$$
\overline{S_{d e t}}=\sum_{i=1}^{\alpha}\left(\begin{array}{c}
\alpha \\
i
\end{array}\right) \frac{L^{i}(N-L)^{\alpha-i}}{N^{a}-(N-L)^{a}} \cdot \frac{\alpha}{i}+\frac{1}{1-\left(\frac{N-L}{N}\right)^{\alpha}}
$$

\section{B. Overall Mean Detection Time}

At each sensing stage, more samples (pre-detection integration) result in a more reliable outcome, i.e., higher detection probability and lower false alarm probability. Therefore, on one hand while the sensing duration is increased, the greater reliability results in fewer number of steps required on average for detecting an available idle channel. Because $M_{c}$ and $M_{f}$ respectively denote the number of sensing samples by CRS and FRS stages, the mean detection time can be written as

$$
\overline{T_{d e t}}=\overline{S_{f r s}}\left(T_{s}+\frac{M_{f}}{B_{c}}\right)+\overline{S_{c r s}}\left(T_{s}+\frac{M_{c}}{\alpha B_{c}}\right)
$$

The total system bandwidth and the bandwidth of a single primary/secondary user is assumed to be $100 \mathrm{MHz}$ and $1 \mathrm{MHz}$ $\left(B_{\text {sys }}=100 \mathrm{MHz}, B_{c}=1 \mathrm{MHz}\right)$, implying that the number of channels is $N=B_{\text {sys }} / B_{c}=100$. Taking different SNR into consideration, we then present the results of sensing duration for noncoherent detector to achieve the performance of $P f_{d}=P c_{d}=0.9, P f_{f a}=P c_{f a}=0.1$ in CRS in Table I, where $T c_{i}$ is the integration time in CRS.

\begin{tabular}{cc|cc|cc|cc}
\hline \hline & & \multicolumn{2}{|c|}{$\mathrm{L} / \mathrm{N}=0.1$} & \multicolumn{2}{c|}{$\mathrm{L} / \mathrm{N}=0.2$} & \multicolumn{2}{c}{$\mathrm{L} / \mathrm{N}=0.3$} \\
\hline$\alpha$ & $\mathrm{SNR}(\mathrm{dB})$ & $M_{c}$ & $T c_{i}(m s)$ & $M_{c}$ & $T c_{i}(m s)$ & $M_{c}$ & $T c_{i}(m s)$ \\
\hline 2 & 3 & 25 & 12.5 & 25 & 12.5 & 24 & 12 \\
4 & 3 & 110 & 27.5 & 100 & 25 & 90 & 22.5 \\
10 & 3 & 600 & 60 & 370 & 37 & 170 & 17 \\
2 & 6 & 11 & 5.5 & 11 & 5.5 & 10 & 5 \\
4 & 6 & 50 & 12.5 & 44 & 11 & 38 & 9.5 \\
10 & 6 & 270 & 27 & 160 & 16 & 70 & 7 \\
2 & 10 & 4 & 2 & 4 & 2 & 4 & 2 \\
4 & 10 & 18 & 4.5 & 16 & 4 & 14 & 4.5 \\
10 & 10 & 100 & 10 & 60 & 6 & 27 & 2.7 \\
\hline
\end{tabular}

TABLE I

SENSING DURATIONS FOR CRS TO ACHIEVE $P f_{d}=P c_{d}=0.9, P f_{f a}=P c_{f a}=0.1$ PERFORMANCE

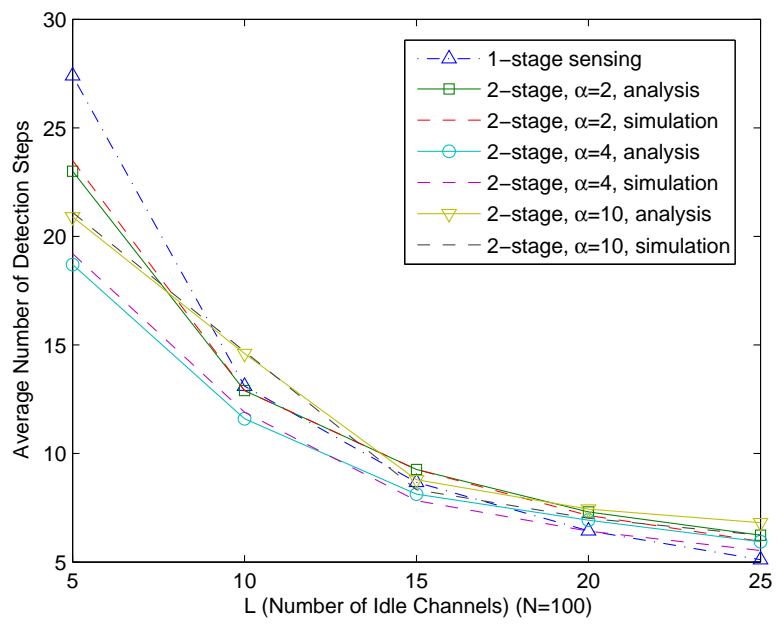

Fig. 3. Average Number of Detection Steps with different values of $\mathrm{L} / \mathrm{N}$ and CSB bandwidth

\section{Numerical Results}

Two-stage sensing and conventional one-stage sensing (random search) are simulated to compare their performance, where $T_{s}$ is assumed to be $20 \mathrm{~ms}$. Matlab is used to generate discrete channels, and the data gathered by running 3000 realizations for each experiment. The average numbers of detection steps of both two sensing schemes are investigated. We set the environment parameters as $P f_{d}=0.9, P f_{f a}=$ $0.05, P c_{d}=0.8, P c_{f a}=0.2, J=4, S N R=3 d B$, and take two-stage sensing with $\alpha=2,4,10\left(B_{c s b}=2,4,10 M H z\right)$ into consideration. It can be noticed from Fig.5 that the simulation results match analysis results well. Another observation is that the system obviously benefits from two-stage sensing when $\mathrm{L} / \mathrm{N}$ is low. As $\mathrm{L} / \mathrm{N}$ goes up, the mean number of detection steps for one-stage sensing sharply decreases, and two-stage sensing loses its advantage due to the excess steps in CRS. Further, the size of CSB's bandwidth also has a tradeoff: small size will incur large number of detection steps for CRS to locate a CSBW; large size will result in the longer time cost in FRS.

To further explore this trade-off, we observe detection performance for different values of CSB bandwidth and $L / N$. The system requirement is $P f_{d}=P c_{d}=0.9, P f_{f a}=P c_{f a}=$ 0.1 . As shown in Fig.6-7, two-stage sensing with $\alpha=2$ outperforms one-stage sensing for $L / N<0.2$. For $\alpha=4$, two-stage mean detection time is lower for $L / N<0.15$. For the same $L$, increasing CSB bandwidth (equivalently $\alpha$ ) implies that larger number of sensing samples are needed to reach the same $P c_{d}-P c_{f a}$ ), thereby leading to increased mean detection time.

Vis-a-vis the impact of varying $L$ : lower the number of idle channels $(L)$, the better performance of two-stage sensing due to faster location of the CSBW. Nonetheless, with the increase in the number of idle channels, the number of detection steps for one-stage sensing sharply decreases quicker than two-stage 


$$
\overline{S_{f r s}}=\left(\overline{S_{f r s, c o r}}+\overline{S_{f r s, f a l}}\right) /\left(1-P_{\text {miss }}\right)=\frac{\sum_{i=1}^{\alpha}\left(\begin{array}{c}
\alpha \\
i
\end{array}\right) \frac{L^{i}(N-L)^{\alpha-i}}{N^{a}-(N-L)^{a}} \frac{(\alpha-i) J P f_{f a}+\alpha}{P f_{d} \cdot i}+\frac{P c_{f a} \alpha\left(1+J P f_{f a}\right)(N-L)^{k}}{P c_{d}\left(N^{k}-(N-L)^{k}\right)}}{1-\sum_{k=1}^{\alpha}\left(\begin{array}{c}
\alpha \\
k
\end{array}\right) \frac{L^{k}(N-L)^{\alpha-k}}{N^{a}-(N-L)^{a}}\left(1-P f_{d}\right)^{k}}
$$

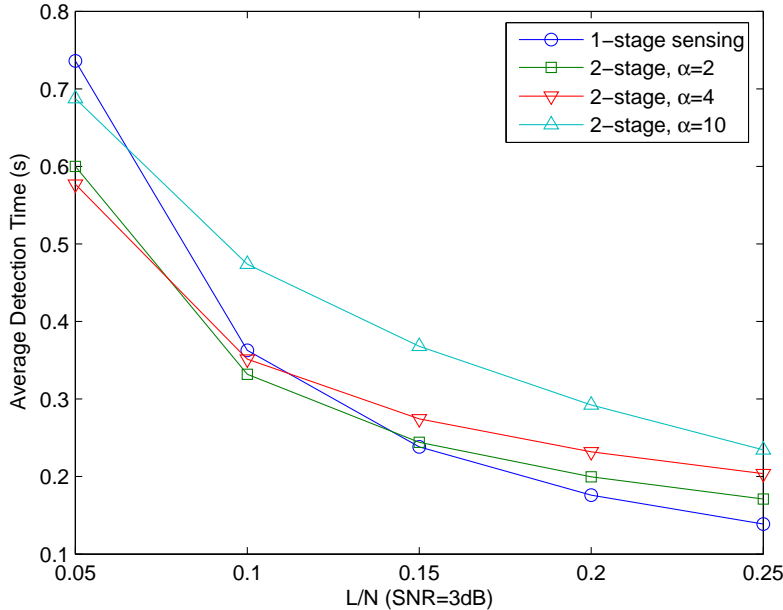

Fig. 4. Mean Detection Time in $\mathrm{SNR}=3 \mathrm{~dB}$ Environment

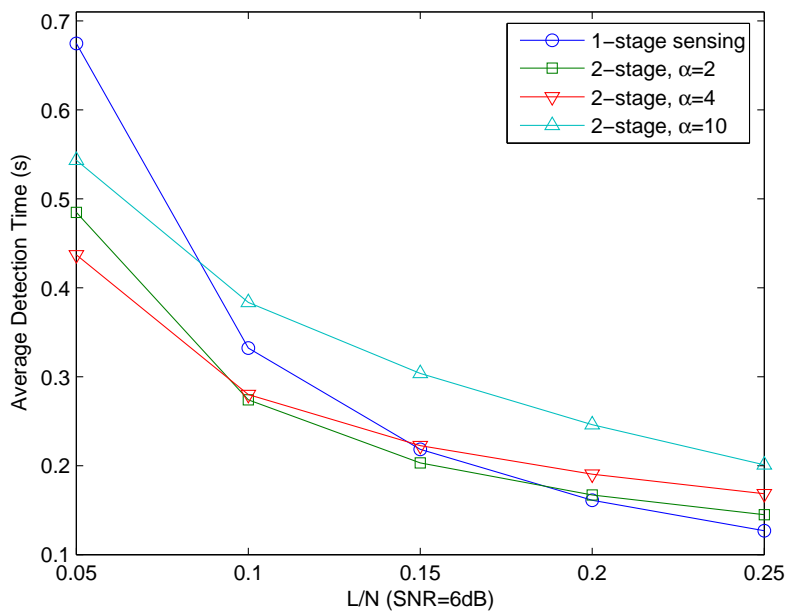

Fig. 5. Mean Detection Time in $\mathrm{SNR}=6 \mathrm{~dB}$ Environment

sensing. Fig. 8 reveals that two-stage sensing with $\alpha=2$ has better performance than one-stage sensing as $L / N<0.2$ : this range increase with increase in SNR. In other words, two-stage sensing is competitive at higher SNRs for larger values of $L$ with one-stage.

\section{CONCLUSION}

In this paper, we presented a new two-stage sensing technique for Dynamic Spectrum Access for enhanced channel sensing. The results show that two-stage sensing with small

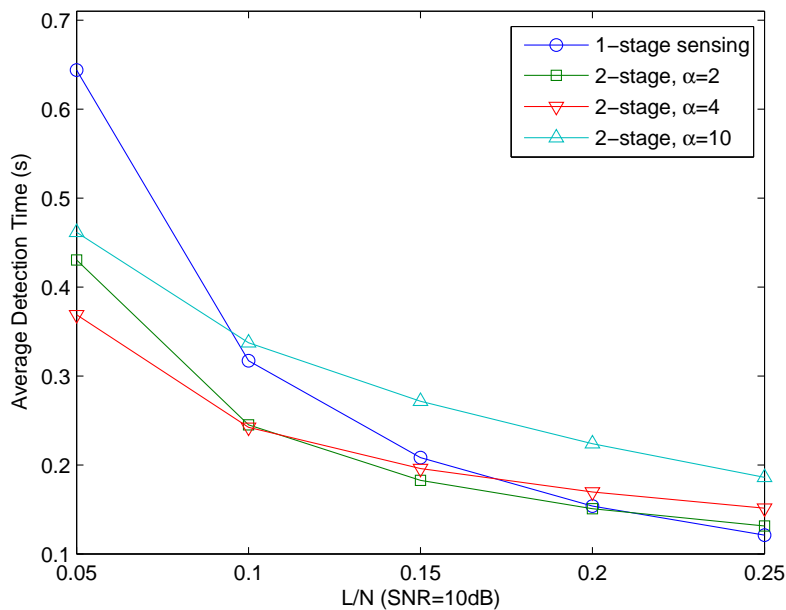

Fig. 6. Mean Detection Time in $\mathrm{SNR}=10 \mathrm{~dB}$ Environment

bandwidth of coarse sensing block outperforms traditional one-stage sensing scheme in terms of lower detection time, when the ratio of idle channels is not high. In particular, system performance trade-offs involving the bandwidth of coarse sensing block and integration time in coarse resolution sensing are also highlighted.

\section{REFERENCES}

[1] FCC, Spectrum Policy Task Force Report, ET Docket No.02-155, Nov. 2002.

[2] M. Nekovee, "Dynamic Spectrum Acess - Concepts and Future Architectures", BT Technology Journal, Vol. 24, No. 2, Apr. 2006.

[3] www.freepress.net/spectrum/whitespace/Seattle.pdf

[4] N.M. Neihart, S. Roy and D.J. Allstot, "A Parallel, Multi-Resolution Sensing Technique for Multiple Antenna Cognitive Radios", IEEE ISCAS 2007, pp. 2530-2533, May 2007.

[5] R.Iyappan and S. Roy, "On Acquisition of Wideband Direct-Sequence Spread Spctrum Signals", IEEE. Trans.on Wireless Commun., Vol. 5, No.6, June 2006.

[6] L. Luo and S. Roy, "Search Schemes and Detection Time Trade-offs for Dynamic Spectrum Access", pre-print.

[7] H. Kim and K.G. Shin, "Adaptive MAC-layer Sensing of Spectrum Availability in Cognitive Radio Networks", Technical Report, CSE-TR518-06, University of Michigan, 2006

[8] G. Ganesan and Y. Li, "Cooperative Spectrum Sensing in Cognitive Radio Networks", IEEE DySPAN 2005, pp. 137-143, Nov. 2005.

[9] C. Cordeiro, K. Challapali and M. Ghosh, "Cognitive PHY and MAC Layers for Dynamic Spectrum Access and Sharing of TV Bands", ACM TAPAS 2006, No. 3, Aug. 2006

[10] A.D. Whalen, Detection of Signals in Noise, Academic Press, 1971.

[11] H. Urkowitz, "Energy Detection of Unknown Deterministic Signals", Proceedings of IEEE, Vol. 55, No. 4, pp. 523-531, Apr. 1997.

[12] R. Iyappan and S. Roy, "Clear Channel Assessment in Energy Constrained Wideband Wireless Networks," IEEE Wireless Commun. Magazine, pp. 70-78, June 2007. 